\title{
MANIFESTAÇÕES EXTRACOLÔNICAS DA POLIPOSE ADENOMATOSA FAMILIAR: incidência e impacto na evolução da doença
}

\author{
Fábio Guilherme CAMPOS, Angelita HABR-GAMA, Desidério Roberto KISS, \\ Fábio César ATUÍ, Fábio KATAYAMA e Joaquim GAMA-RODRIGUES
}

RESUMO - Racional - A polipose adenomatosa familiar é doença hereditária de caráter autossômico dominante, que freqüentemente se associa a numerosas manifestações extracolônicas. Objetivos - Relatar a incidência de manifestações extracolônicas em nosso meio e analisar seu impacto na evolução da doença. Pacientes e Métodos - Revisão dos prontuários de pacientes com polipose adenomatosa familiar tratados no período de 1977 a 2001, relatando as manifestações extracolônicas associadas e suas complicações. Resultados Dos 59 pacientes com polipose adenomatosa familiar, 23 (38,9\%) apresentaram alguma manifestação extracolônica por ocasião do diagnóstico ou no seguimento. Foram registradas 37 diferentes manifestações (1,6 por paciente). As mais comuns foram osteomas e alterações na pigmentação da retina, diagnosticadas em $25 \%$ e $20 \%$ dos pacientes pesquisados, respectivamente. Outras manifestações extracolônicas achadas foram adenomas do trato digestivo superior, cistos epidermóides, tumores desmóides (sete cada), câncer gástrico (três) e câncer de tireóide (dois). Complicações importantes diretamente relacionadas aos tumores desmóides foram reportadas em seis pacientes, sendo obstrução intestinal em quatro e hidronefrose em dois. Registraram-se óbitos em dois pacientes (28,5\%). Conclusões - A incidência de manifestações extracolônicas é alta (40\%), podendo afetar a evolução da doença e a qualidade de vida dos pacientes. Por esses motivos, torna-se de fundamental importância a pesquisa, a prevenção e o tratamento adequado dessas manifestações na polipose adenomatosa familiar.

DESCRITORES - Polipose adenomatosa do cólon. Síndrome de Gardner. Adenoma.

\section{INTRODUÇÃO}

Descrita por MENZELIO ${ }^{(28)}$ em 1721, a polipose adenomatosa familiar (PAF) é doença hereditária de caráter autossômico dominante, sendo responsável por apenas $1 \%$ dos casos de câncer colorretal (CCR) na população ${ }^{(17)}$. O defeito genético localiza-se no gene APC (adenomatous polyposis coli) situado no braço longo do cromossomo $5 \mathrm{q} 21^{(22)}$. Em aproximadamente $20 \%$, a doença pode ser decorrente de mutações genéticas ${ }^{(14)}$.

Do ponto de vista clínico, a doença geralmente se manifesta na puberdade, com o aparecimento de pólipos adenomatosos na mucosa colorretal ${ }^{(5)}$. Sintomatologia associada geralmente sucede $\mathrm{o}$ aparecimento dos adenomas na segunda década de vida. A natureza adenomatosa e a enorme quantidade de pólipos tornam a possibilidade de degeneração maligna preocupação importante em pacientes não tratados, em que o desenvolvimento de CCR é regra, surgindo em média 10 anos após o desenvolvimento dos pólipos $^{(43)}$.

Filhos de indivíduo afetado têm risco de $50 \%$ de herdar o gene. Após a identificação do paciente chamado "índice", a realização de teste genético facilita a identificação mais acurada dos familiares eventualmente acometidos, permitindo focar o rastreamento e o tratamento profilático somente naqueles com risco ${ }^{(21)}$. Desta forma, assume fundamental importância a detecção precoce de pacientes com esta afecção, seu adequado tratamento pela colectomia profilática e o rastreamento de familiares para identificar eventuais portadores do defeito genético.

Desde a descrição original de polipose associada a cistos epidermóides e osteoma, a combinação de PAF e manifestações extracolônicas (MEC) é comumente referida como síndrome de Gardner ${ }^{(11)}$. Subseqüentemente, reconheceu-se que a PAF é uma pan-polipose gastrointestinal que pode estar associada a numerosas MEC, benignas e malignas.

Disciplina de Coloproctologia do Departamento de Gastroenterologia da Faculdade de Medicina da Universidade de São Paulo.

Endereço para correspondência: Dr. Fabio Campos - Alameda Jaú, 1477 - apto 111A - 01420-002 - São Paulo, SP. e-mail: fgmcampos@terra.com.br 
Além do cólon e reto, pólipos podem ser encontrados também no trato digestivo superior (estômago e duodeno), intestino delgado, tireóide, adrenais, pâncreas e hipófise ${ }^{(6)}$. Outras MEC benignas incluem cistos sebáceos, lipomas, osteomas, dedos hipocráticos, anormalidades dentárias (dentes supranumerários), lesões da retina e tumores desmóides. Entre as manifestações malignas, foram reportados tumores na região periampular, de ductos biliares, gástricos, no íleo (carcinoma e carcinóide), tireóide, supra-renal e sistema nervoso central (Figura 1).

FIGURA 1 - Incidência das manifestações extracolônicas da polipose adenomatosa familiar reportadas na literatura

\begin{tabular}{|lc|}
\hline Manifestações & Incidência (\%) \\
\hline Cistos epidermóides & 50 \\
Osteomas & $14-93$ \\
Tumor desmóide & $4-29$ \\
Tumor de intestino delgado & raro \\
CHRPE & $58-92$ \\
Tumor hepatobiliar & $<1$ \\
Tumor do SNC & raro \\
Tumor de tireóide & Risco em mulheres 20-160 vezes \\
& $>$ população geral \\
\hline Polipose de glândulas fúndicas & $23-56$ \\
Pólipos hiperplásicos & $8-44$ \\
Adenoma gástrico & $2-13$ \\
Carcinoma gástrico & $<1$ \\
Adenoma duodenal & $24-100$ \\
Carcinoma duodenal & Desconhecido 50-300 vezes \\
& $>$ população geral \\
\hline
\end{tabular}

Como a doença não é confinada ao cólon e as MEC, podem ser causa importante de morbimortalidade, torna-se importante diagnosticar, prevenir e tratar essas manifestações ${ }^{(34,37,43)}$.

Assim, o presente trabalho visou relatar a incidência de MEC em nosso meio e analisar seu impacto na evolução da doença em pacientes portadores de PAF.

\section{PACIENTES E MÉTODOS}

A população deste estudo foi constituída por pacientes com PAF atendidos no Serviço de Coloproctologia do Hospital das Clínicas da Faculdade de Medicina da Universidade de São Paulo. Foram revisados retrospectivamente os prontuários de pacientes atendidos no período de 1977 a 2001 .

Levantaram-se dados relativos a sexo e idade por ocasião do diagnóstico da PAF, tipos e localização das manifestações extracolônicas, suas repercussões na evolução da doença, dados sobre seu tratamento e resultados.

\section{RESULTADOS}

No período foram tratados 59 pacientes portadores de PAF, sendo 32 mulheres (54,2\%) e 27 homens (45,8\%). A idade variou de 7 a 68 anos (média de 34,1 anos).

Desse total, 23 pacientes (38,9\%) apresentaram algum tipo de MEC por ocasião do diagnóstico ou ao longo da evolução da doença. Foram registradas 37 diferentes MEC, totalizando a relação de 1,6 MEC por paciente com PAF (Tabela 1).

TABELA 1 - Tipo e incidência de manifestações extracolônicas diagnosticadas

\begin{tabular}{lcc}
\hline Manifestação extracolônica & Número & \% \\
\hline Cistos epidermóides & 7 & 11,9 \\
Pigmentação da retina & $5 / 25^{*}$ & 20 \\
Osteomas & $6 / 24^{*}$ & 25 \\
Tumores desmóides & 7 & 11,9 \\
Câncer gástrico & 3 & 5,1 \\
Câncer de tireóide & 2 & 3,4 \\
Adenoma gástrico & 5 & 8,5 \\
Adenoma duodenal & 2 & 3,4 \\
Total & $\mathbf{3 7}$ & $\mathbf{1 , 6}$ por paciente \\
\hline
\end{tabular}

* A fração representa número de achados sobre pacientes pesquisados

Como se pode observar nesta tabela, as manifestações mais comuns foram osteomas e alterações na pigmentação da retina (CHRPE), diagnosticadas em $25 \%$ e $20 \%$ dos pacientes pesquisados, respectivamente. Entre os pacientes com CHRPE, apenas um desenvolveu também osteoma de mandíbula.

Foram diagnosticados adenomas no trato digestório superior em sete pacientes. Os pacientes com pólipos adenomatosos no estômago (cinco) foram submetidos a polipectomia endoscópica em períodos que variaram de 9 a 135 meses após o diagnóstico da polipose. Um deles perdeu o seguimento e desenvolveu câncer gástrico com metástase hepática, falecendo 7 meses após. Este paciente tinha sido submetido a proctocolectomia total com ileostomia definitiva por câncer de reto. Dois pacientes desenvolveram pólipos duodenais, que foram ressecados com 12 e 30 meses de seguimento.

Dois outros pacientes desenvolveram câncer gástrico e foram tratados por gastrectomia total 126 e 130 meses após o tratamento da PAF. Esses pacientes tinham pólipos adenomatosos no estômago por ocasião do diagnóstico de polipose. Os pacientes com câncer gástrico tinham 49, 59 e 60 anos de idade.

Ambas as pacientes que desenvolveram câncer de tireóide eram do sexo feminino, com idades de 20 e 24 anos por ocasião deste diagnóstico. Foram submetidas a tireoidectomia total 9 e 24 meses após o diagnóstico de PAF, respectivamente. Essas pacientes estão vivas sem doença 36 e 96 meses após tratamento. 
Tumores desmóides ocorreram em sete pacientes (12\%), sendo cinco mulheres e dois homens em idades que variaram de 21 a 60 anos (média de 35,8 anos). Desses pacientes, seis haviam sido submetidos à colectomia prévia em períodos que variaram de 21 a 60 meses. Em apenas um paciente diagnosticou-se tumor retroperitonial simultaneamente ao diagnóstico da polipose.

Quanto à localização, os tumores estavam localizados no mesentério (quatro), parede abdominal (um), retroperitônio (um) e parede abdominal + retroperitônio (um). Complicações importantes diretamente relacionadas aos tumores desmóides foram reportadas em seis pacientes, sendo obstrução intestinal em quatro e hidronefrose em dois. Ocorreram óbitos em dois pacientes $(28,5 \%)$.

\section{DISCUSSÃO}

A PAF constitui síndrome genética de caráter dominante, caracterizada por maior predisposição em desenvolver CCR e outras lesões extracolônicas. Está associada a mutações germinativas no gene APC que codifica uma proteína com 2843 aminoácidos, com funções na regulação do crescimento e proliferação celular, gerando assim o desenvolvimento de lesões benignas e malignas em diferentes órgãos ${ }^{(23)}$.

A PAF e a síndrome de Gardner se originam de mutações no mesmo gene, sem alteração na localização ou natureza das mutações, mas com expressões fenotípicas variáveis (Figura 2). A associação de polipose com tumores do sistema nervoso central é conhecida como síndrome de Turcot $^{(39)}$. Nesta síndrome, descreveram-se mutações no gene APC (dando origem a tumores do tipo meduloblastoma e astrocitoma) e nos genes de reparo associados ao HNPCC (dando origem a glioblastomas) $)^{(4,40,42)}$.

Aproximadamente $96 \%$ das mutações na PAF determinam a formação de uma proteína truncada, que é determinada pela realização do teste de proteína truncada ou IVSP (in vitro synthesizedprotein assay $)^{(31)}$. Quando se localiza a proteína truncada, é possível localizar a mutação em um segmento específico do gene e, a seguir, realizar o seqüenciamento genético para identificar um ou mais nucleotídeos mutantes.
Parece haver relação entre local da mutação e expressões fenotípicas diferentes. Assim, mutações localizadas no final 5' do gene determinam o aparecimento de fenótipo brando caracterizado por menor número de adenomas, progressão mais lenta e aparecimento tardio da doença. Já mutações no final 3' se relacionam com fenótipo colônico variável e maior chance de manifestações extracolônicas. Enquanto mutações nos códons 1061 e 1369 predispõem à polipose típica, aquelas localizadas no códon 1465 estão associadas a expressões fenotípicas variáveis no cólon e outros órgãos ${ }^{(15)}$.

Os tumores de partes moles (cistos epidermóides e fibromas) podem ocorrer em qualquer lugar da superfície cutânea, predominando nos membros, face e couro cabeludo. Na presente casuística, foram diagnosticados cistos em $12 \%$ dos pacientes. LEPPARD e BUSSEY ${ }^{(25)}$ encontraram cistos em 53\% de 70 PAF.

Embora tenham apenas interesse cosmético, sua importância reside no fato de que são lesões raras na infância em indivíduos sem polipose; além disso, osteomas e tumores de partes moles podem preceder o aparecimento de pólipos intestinais.

As MEC mais comumente diagnosticadas são as lesões da retina, originalmente interpretadas como congênitas (hipertrofia congênita do epitélio pigmentar da retina, cuja sigla em inglês é CHRPE), que podem aparecer em até $90 \%$ dos parentes de um portador de $\mathrm{PAF}^{(41)}$. Embora achados histopatológicos indiquem que o termo "hamartomas do epitélio pigmentar da retina" seja mais apropriado para designar essas lesões, o termo CHRPE é o mais aceito e continua a ser usado ${ }^{(6,33)}$.

Alguns autores sugeriram que a existência de quatro ou mais lesões CHRPE distribuídas am ambos os olhos seria um marcador fenotípico da polipose que, quando diagnosticado em determinada família, também seria encontrado em todos os pacientes do mesmo grupo familiar. Desta forma, a CHRPE poderia facilitar a detecção de mutações constitucionais do APC em parentes ${ }^{(19,21)}$.

BERTARIO et al. ${ }^{(1)}$ observaram que a sensibilidade do CHRPE para PAF era de $70 \%$, com valor preditivo de $92 \%$. Ressaltaram, porém, que a ausência de lesões na retina não elimina a necessidade de seguimento adequado de indivíduos com risco. Em 1996, ROSSATO et al. ${ }^{(33)}$ constataram que $43,7 \%$ dos indivíduos com áreas de CHRPE

FIGURA 2 - Síndromes relacionadas à polipose adenomatosa familiar

\begin{tabular}{|lll|}
\hline Síndrome & Gene e mecanismo & Tumores associados \\
\hline PAF e Gardner & $\begin{array}{l}\text { APC } \\
\text { Autossômico dominante }\end{array}$ & $\begin{array}{l}\text { Desmóides, periampular, } \\
\text { duodenal, gástrico, } \\
\text { intestino delgado } \\
\text { PAF atenuada }\end{array}$ \\
Turcot & $\begin{array}{l}\text { APC } \\
\text { Autossômico dominante }\end{array}$ \\
& $\begin{array}{l}\text { APC e genes de } \\
\text { reparo do DNA } \\
\text { Autossômico recessivo }\end{array}$ & Cérebro \\
\hline
\end{tabular}

PAF $=$ polipose adenomatosa familiar

$\mathrm{APC}=$ "adenomatous polyposis coli" 
também tinham polipose, enquanto $58,3 \%$ dos indivíduos com PAF tinham CHRPE. Esta alteração também foi diagnosticada em 5,5\% de um grupo-controle normal. Por outro lado, outros encontraram CHRPE em $100 \%$ das poliposes ${ }^{(24)}$. Nesta casuística, observaram-se alterações da retina em $20 \%$ dos pacientes submetidos a exame oftalmológico.

A CHRPE tem sido consistentemente associada a determinado domínio (códons 463 a 1387) e é o único fenótipo sem variação intra-familiar ${ }^{(7)}$. Dessa maneira, a caracterização de CHRPE pode adicionar informação sobre a localização da mutação genética ${ }^{(34)}$. Entretanto, as inter-relações entre análise mutacional, manifestações clínicas e decisões terapêuticas específicas ainda carecem de futuras investigações.

Até o momento, reconhece-se que o achado de lesões CHRPE ao exame oftalmológico é um marcador clínico a mais para PAF em famílias CHRPE "positivas". Em famílias CHRPE "negativas”, exames oftálmicos negativos não têm valor profético e não devem eliminar a pessoa de rastreamento futuro.

Tumores desmóides (TD) e lesões da retina (CHRPE) segregamse independentemente de outras MEC. Em pacientes com PAF, as principais causas de mortalidade são os TD, as neoplasias colorretais e periampulares ${ }^{(2)}$.

Apesar de histologicamente benignos (lesões fibromatosas), os TD podem exibir comportamento biológico agressivo, com invasão local, mas não metastatizam. Formam tumores encapsulados, de crescimento lento, podendo surgir no mesentério, parede abdominal, incisões, retroperitônio, virilha e nádegas; eventualmente regridem espontaneamente e podem ser multifocais ${ }^{(26,30,38,43)}$.

A incidência pós-operatória desses tumores varia de 3,5\% a 29\% (com média entre 10\% e 18\%), ocorrendo geralmente cerca de 2 anos após a cirurgia colorretal ${ }^{(2,16,18,20,30)}$. Entretanto, o número de diagnósticos fortuitos em pacientes assintomáticos sugere que esta incidência é provavelmente maior do que a reportada.

A ocorrência desses tumores está associada a diferentes fatores de risco. Cerca de $80 \%$ dos casos ocorrem em pacientes submetidos a operações abdominais prévias. No presente levantamento, TD foram diagnosticados em sete pacientes (12\%), seis dos quais submetidos a tratamento cirúrgico em períodos que variaram de 20 a 60 meses. Desses pacientes, cinco eram mulheres com idades que variaram de 21 a 60 anos (média: 35,8 anos).

Na Mayo Clinic, LOTFI et al. ${ }^{(26)}$ reportaram TD mesentéricos em $13 \%$ de 183 pacientes com PAF; a média etária foi de 31 anos em mulheres e 37 anos entre os homens. IWAMA et al. ${ }^{(20)}$ também observaram predomínio no sexo feminino entre os 71 TD $(6,7 \%)$ diagnosticados entre 1050 PAF com idade média de 32 anos.

Sexo feminino, história familiar de desmóides, presença de osteomas e mutações além do códon 1444 são considerados fatores preditivos independentes ${ }^{(2,30)}$. História familiar de desmóide tem sido reportada em mais de $50 \%$ dos portadores ${ }^{(16,27)}$. Sua incidência também parece estar associada a mulheres na pré-menopausa, gravidez e uso de contraceptivos. Essas observações indicam que a integração entre dados clínicos e genéticos ajuda a definir subgrupos de pacientes com maior risco para desenvolver esses tumores.

Quando pequenos, os TD podem ser assintomáticos. À medida que crescem, podem determinar sintomas relacionados a complicações como compressão ureteral, obstrução intestinal, infilltração de outros órgãos, fístulas e oclusão vascular ${ }^{(13,30,35)}$. Morte sobrevém em média 6 anos após o diagnóstico ${ }^{(16)}$.

Entre os TD tratados, a maioria estava localizada no mesentério (quatro) e retroperitônio (dois). Esses pacientes desenvolveram obstrução intestinal e hidronefrose, levando a óbito dois deles (28,5\%). Embora a manipulação cirúrgica mínima possa determinar menos complicações e menor risco de desmóides, a prevalência desses tumores não é maior em pacientes submetidos a bolsa ileal, em comparação a outros procedimentos ${ }^{(10,30)}$.

As opções para tratamento incluem cirurgia, radioterapia e quimioterapia com drogas citotóxicas e não-citotóxicas. O manuseio dos TD representa um grande desafio. A ressecção cirúrgica completa é raramente possível em TD mesentéricos devido à extensão da ressecção e ao alto risco de recidiva. Dessa maneira, a cirurgia deve ser reservada para alívio da obstrução, preferindo-se procedimentos de "bypass" a de ressecção(26, 30).

Terapias não-cirúrgicas têm sido empregadas em séries com pequeno número de pacientes. $\mathrm{O}$ manuseio dos TD com drogas não-citotóxicas, como antiinflamatórios não-esteróides (sulindac, indometacina) e antiestrogênicos (tamoxifen, toremifene, progesterona) tem propiciado resultados muitas vezes variados. Entretanto, reportaram-se benefícios evidentes como redução do tamanho e da sintomatologia, em comparação a grupos de TD não tratados ${ }^{(38)}$.

A indicação de radioterapia é questionada pela resposta limitada e devido às potenciais complicações como enterite actínica. Apesar da eficácia ainda não devidamente comprovada, quimioterápicos têm sido indicados seletivamente em tumores sintomáticos irressecáveis que não respondem a outras terapias ${ }^{(30)}$.

Pólipos gastroduodenais são freqüentemente observados na PAF, e mais raramente no intestino delgado. As lesões gástricas mais encontradas são pólipos de glândulas fúndicas (dilatações císticas), hiperplásicos e, menos freqüentemente, adenomas e carcinoma. De acordo com a literatura japonesa, a incidência de carcinoma gástrico na PAF varia de $4,5 \%$ a $13,6 \%$, enquanto fora do Japão esta é uma complicação rara ${ }^{(20)}$.

No presente estudo diagnosticaram-se adenomas gástricos em cinco pacientes $(8,5 \%)$ e duodenais em dois $(3,4 \%)$. Três pacientes $(5,1 \%)$ foram tratados por câncer gástrico, sendo dois deles submetidos a gastrectomia total e outro perdeu seguimento, morrendo com metástase hepática.

Por outro lado, adenomas duodenais são diagnosticados em até $90 \%-100 \%$ dos pacientes ao longo do seguimento ${ }^{(6,36)}$, sendo os 
adenomas periampulares (muitas vezes microscópicos) as lesões precursoras de carcinoma nesta região.

Estima-se que pacientes com PAF tenham chance 300 vezes maior que a população geral para desenvolver câncer gastroduodenal ${ }^{(9)}$. O carcinoma periampular é a forma mais comum de câncer extracolônico, com risco em vida estimado em $10 \%-12 \%$ e é responsável por grande número de mortes por câncer pós-colectomia total ${ }^{(4,29)}$.

NUGENT et al. ${ }^{(29)}$ estimaram que a probabilidade cumulativa de um paciente desenvolver câncer extracolônico aumenta com a idade, sendo de $11 \%$ aos 50 anos e $52 \%$ aos 75 anos, números significativamente superiores aos da população geral. Quase metade desses tumores era periampular em sua origem.

Adenomas do intestino delgado foram reportados no íleo terminal, na mucosa ileal pós-ileostomia, íleo-reto anastomose, bolsa ileal e bolsa de Koch. A transformação maligna desses pólipos é rara ${ }^{(20)}$.

Embora ainda não tenham sido definidos programas de vigilância endoscópica e tratamento efetivo para essas lesões, sabe-se que o uso de antiinflamatórios não-esteróides como sulindac não controla os pólipos $^{(32)}$. IWAMA et al. ${ }^{(20)}$ propuseram ressecção local da papila de Vater em pacientes acima de 35 anos com adenoma.

Em revisão de 1.050 portadores de PAF, IWAMA et al. ${ }^{(20)}$ encontraram 23 carcinomas duodenais, 27 gástricos, 11 de tireóide e 71 TD. Já foram também reportados tumores hepatobiliares, pancreáticos, de bexiga, rins, testículos, olhos e pulmões. Esses autores observaram que carcinomas da tireóide se desenvolvem em idade menor (32 anos) que tumores do TGI (43 anos em duodeno e 49 anos no estômago) e que mulheres apresentam maior propensão para desenvolver carcinoma de tireóide e TD. Estima-se que o risco de carcinoma da tireóide associado à PAF seja 100 a 160 vezes superior ao da população geral em mulheres européias ${ }^{(3)}$, contra 25 vezes no Japão ${ }^{(20)}$.

No presente levantamento, encontrou-se câncer de tireóide em duas pacientes do sexo feminino (3,4\%). Essas pacientes tinham $20 \mathrm{e}$ 24 anos, respectivamente; por outro lado, a idade média dos três pacientes com câncer gástrico foi maior (56 anos).

Em 15 pacientes com carcinoma papilar da tireóide associada à PAF, CETTA et al. ${ }^{(8)}$ observaram que a mutação estava localizada entre os códons 778 e 1309 (exon 15) em 13 pacientes, documentando manchas oculares em 12 pacientes. Dessa maneira, as mutações agruparam-se na área genômica associada à CHRPE (códons 463-
1387). Esses autores sugeriram que a incidência de câncer tireoideano foi subestimada no passado e que o rastreamento intensivo poderia detectar maior número desses tumores. Recomendam ainda pesquisa sistemática em pacientes com manchas oculares e mutações genéticas no exon 15, destacando que esses tumores parecem ter excelente prognóstico.

Os osteomas foram primeiramente descritos por GARDNER e RICHARDS ${ }^{(12)}$, em 1953. Podem ocorrer em qualquer osso, embora sejam mais comuns na face (particularmente no ângulo da mandíbula) e menos freqüentes em ossos frontais e occipitais. São tumores benignos, embora possam causar sintomas por crescimento local. Já foram descritos dois casos de sarcoma osteogênico ${ }^{(21)}$.

Ocasionalmente aparecem antes do diagnóstico da polipose, podendo sugerir a herança do gene em descendentes de indivíduos afetados. A incidência relatada (14\% a 93\%) é bastante variável, refletindo diferenças na assiduidade com que se procura tais lesões, na interpretação das radiografias e na idade em que são pesquisadas ${ }^{(6)}$. No presente trabalho, diagnosticaram-se osteomas em $25 \%$ dos pacientes em que se investigou esta manifestação.

\section{CONCLUSÕES}

Os dados aqui apresentados e a revisão da literatura pertinente sugerem que a incidência de MEC ao longo da evolução da PAF é alta, tendo sido encontradas em quase $40 \%$ dos pacientes tratados. Devese ressaltar que, provavelmente, este número possa ser ainda maior, na dependência da realização de pesquisa rotineira dessas alterações e de seguimento prolongado.

Constata-se que algumas dessas manifestações (especialmente as neoplásicas) podem trazer graves conseqüências e gerar importantes complicações, afetando o tempo e a qualidade de vida. Por esses motivos, torna-se necessário conhecer e investigar essas manifestações não só por ocasião do diagnóstico da PAF, como também no seguimento pósoperatório desses pacientes.

\section{AGRADECIMENTOS}

Aos Drs. Afonso Henrique da Silva e Sousa Jr. e José Hyppólito da Silva que também participaram do atendimento ambulatorial dos pacientes. 
Campos FG, Habr-Gama A, Kiss DR, Atuí FC, Katayama F, Gama-Rodrigues J. Extracolonic manifestations of familial adenomatous polyposis: incidence and impact on the disease outcome. Arq Gastroenterol 2003;40(2):92-98.

ABSTRACT - Background - Familial adenomatous polyposis is a hereditary disease with autossomic and dominant features, frequently associated to many extracolonic manifestations. Aim - To report extracolonic manifestations incidence and to analyze its impact on the disease's outcome. Patients and Methods - Revision of patient charts treated from 1977 to 2001, relating associated extracolonic manifestations and its complications. Results - Among 59 familial adenomatous polyposis patients, 23 (38,9\%) presented some extracolonic manifestations at diagnosis or during follow-up. There were registered 37 different extracolonic manifestations (1.6 per patient). The most common manifestations were osteomas and congenital hypertrophy of the retinal pigment epithelium, diagnosed in $25 \%$ and $20 \%$ of the investigated patients, respectively. Other extracolonic manifestations were represented by upper digestive adenomas, epidermoid cysts, desmoid tumor (seven each), gastric cancer (three) and tireoid cancer (two). Desmoid-associated complications were reported in six patients, being intestinal obstruction in four and hidronephrosis in two; two deaths $(28,5 \%)$ were registered. Conclusions - Extracolonic manifestations incidence is high $(40 \%)$ and may affect disease's outcome and patient's quality of life. For these reasons, the research, prevention and adequate treatment of extracolonic manifestations turn into vital importance in familial adenomatous polyposis patients.

HEADINGS - Adenomatous polyposis coli. Gardner syndrome. Adenoma.

\section{REFERÊNCIAS BIBLIOGRÁFICAS}

1. Bertario L, Bandello F, Rossetti C, Sala P, Fortini E, Spinelli P, Gennari L, Pietroiusti M, Presciuttini S. Congenital hypertrophy of retinal pigment epithelium (CHRPE) as a marker for familial adenomatous polyposis (FAP). Eur J Cancer Prev 1993;2:69-75.

2. Bertario L, Russo A, Sala P, Eboli M, Giarola M, D'amico F, Gismondi V, Varesco L, Pierotti MA, Radice P, Hereditary Colorectal Tumours Registry. Genotype and phenotype factors as determinants of desmoid tumors in patients with familial adenomatous polyposis. Int J Cancer 2001;95:102-7.

3. Büllow S. Clinical features in familial polyposis coli. Dis Colon Rectum 1986;29:102-7.

4. Burt RW. Familial risk and colorectal cancer [review]. Gastroenterol Clin North Am 1996;25:793-803

5. Bussey HJR. Familial polyposis coli; family studies, histopathology, differentia diagnosis and results of treatment. Baltimore: Johns Hopkins University; 1975. p.59-63.

6. Campbell WJ, Spence RA, Parks TG. Familial adenomatous polyposis. Br J Surg 1994;81:1722-33

7. Cao X, Eu KW, Seow-Choen F, Zao Y, Cheah PY. APC mutation and phenotypic spectrum of Singapore familial adenomatous polyposis patients. Eur J Hum Genet $2000 ; 8: 42-8$.

8. Cetta F, Olschwang S, Petracci M, Montalto G, Baldi C, Zuckermann M, Costantin RM, Fusco A. Genetic alterations in thyroid carcinoma associated with familial adenomatous polyposis: clinical implications and suggestions for early detection. World J Surg 1998;22:1231-6.

9. Dean PA. Hereditary intestinal polyposis syndromes. Rev Gastroenterol Méx 1996;61:100-11.

10. Dozois RR, Kelly KA, Welling DR, Gordon H, Beart RW Jr, Wolff BG, Pemberton $\mathrm{JH}$, Ilstrup DM. Ileal pouch-anal anastomosis: comparison of results in familia adenomatous polyposis and chronic ulcerative colitis. Ann Surg 1989;210:268-71.

11. Gardner EJ. A genetic and clinical study of intestinal polyposis: a predisposing factor for carcinoma of the colon and rectum. Am J Genet 1951;3:167-76.

12. Gardner EJ, Richards RC. Multiple cutaneous and subcutaneous lesion occurring simultaneously with hereditary intestinal polyposis and osteomas. Am J Hum Genet 1953;5:139-47.

13. Griffioen G, Bus PJ, Vasen HF, Verspaget HW, Lamers CB. Extracolonic manifestations of familial adenomatous polyposis: desmoid tumours, and upper gastrointestinal adenomas and carcinomas [review]. Scand J Gastroentero 1998;225:85-91.

14. Groden J, Thliveris A, Samowitz W, Carlson M, Gelbert L, Albertsen H, Joslyn G, Stevens J, Spirio L, Robertson M, Sargeant L, Krapcho K, Wolff E, Burt R, Hughes JP, Warrington J, McPherson J, Wasmuth J, Le Paslier D, Abderrahim H, Cohen D, Leppert M, White R. Identification and characterization of the familial adenomatous polyposis coli gene. Cell 1991;66:589.

15. Guldenschuh I, Hurliman R, Muller A, Ammann R, Mullhaupt B, Dobbie Z, Zala GF, Flury R, Seelentag W, Roth J, Meyenberger C, Fried M, Hoppeler T, Spigelman AD, Scott RJ. Relationship between APC genotype, polyp distribution, and oral sulindac treatment in the colon and rectum of patients with familial adenomatous polyposis. Dis Colon Rectum 2001;44:1090-9.

16. Gurbuz AK, Giardiello FM, Petersen GM, Krush AJ, Offerhaus GJA, Booker SV, Kerr MC, Hamilton SR. Desmoid tumors in familial adenomatous polyposis. Gut 1994;35;377-81

17. Habr-Gama A, Campos FG. Síndromes polipóides do intestino grosso. In: Pinotti HW, editor. Tratado de clínica cirúrgica do aparelho digestivo. São Paulo: Atheneu; 1994. p. 1247-58

18. Heiskanen I, Jarvinen HJ. Occurrence of desmoid tumours in familial adenomatous polyposis and results of treatment. Int J Colorectal Dis 1996;11:157-62.

19. Heyen F, Jagelman DG, Romania A, Zakov ZN, Lavery IC, Fazio VW, McGannon E. Predictive value of congenital hypertrophy of the retinal pigment epithelium as a clinical marker for familial adenomatous polyposis. Dis Colon Rectum 1990;33:1003-8.

20. Iwama T, Mishima Y, Utsunomiya J. The impact of familial adenomatous polyposis on the tumorigenesis and mortality at the several organs. Its rational treatment. Ann Surg 1993;217:101-8.

21. Jagelman DG. Familial polyposis coli. In: Fazio VW, editor. Current therapy in colon and rectal surgery. Philadelphia, PA: Decker; 1990. p. 284-8.

22. Kinzler KW, Nilbert MC, Su L, Su LK, Vogelstein B, Bryan TM, Levy DB, Smith KJ, Preisinger AC, Hedge P, McKechnie D. Identification of FAP locus genes from chromosome 5q21. Science 1991;253:661-4.

23. Lal G, Gallinger S. Familial adenomatous polyposis. Semin Surg Oncol 2000;18:314-23

24. Lam DS, Kwok SP, Kwok AK, Liew CT, Lau JW, Pang CC. Incidence and predictive value of congenital hypertrophy of retinal pigment epithelium in Chinese familial adenomatous polyposis patients. Chin Med J (Engl) 1998;111:278-81.

25. Leppard B, Bussey HJR. Epidermoid cysts, polyposis coli and Gardner's syndrome. Br J Surg 1975;62:387-93.

26. Lotfi AM, Dozois RR, Gordon H, Hruska LS, Weiland LH, Carryer PW, Hurt RD. Mesenteric fibromatosis complicating familial adenomatous polyposis: predisposing factors and results of treatment. Int J Colorectal Dis 1989;4:30-6.

27. McAdam WA, Goligher JC. The occurrence of desmoids in patients with familial polyposis. Br J Surg 1970:57:618-31.

28. Menzelio D. De excrescentals verrucosa cristois in intestinis crassis dysenteriam passi observatis. Acta Medicorum Berolinensium 1721;4:68-71.

29. Nugent KP, Spigelman AD, Phillips RKS. Risk of extracolonic cancer in familial adenomatous polyposis. Br J Surg 1996;83:1121-2.

30. Penna C, Tiret E, Parc R, Sfairi A, Kartheuser A, Hannoun L, Nordlinger B. Operation and abdominal desmoid tumors in familial adenomatous polyposis. Surg Gynecol Obstet 1993;177:263-8.

31. Rabelo R, Foulkes W, Gordon PH, Wong N, Yuan ZQ, MacNamara E, Chong G Pinsky L, Lasko D. Role of molecular diagnostic testing in familial adenomatous polyposis and hereditary nonpolyposis colorectal cancer families. Dis Colon Rectum 2001;44:437-46.

32. Richard CS, Berk T, Bapat BV, Haber G, Cohen Z, Gallinger S. Sulindac for periampullary polyps in FAP patients. Int J Colorectal Dis 1997;12:14-8.

33. Rossato M, Rigotti M, Grazia M, Turco AE, Bonomi L. Congenital hypertrophy of the retinal pigment epithelium (CHRPE) and familial adenomatous polyposis (FAP). Acta Ophthalmol Scand 1996;74:338-42. 
34. Ruhswurm I, Zehetmayer M, Dejaco C, Wolf B, Karner-Hanusch J. Ophthalmic and genetic screening in pedigrees with familial adenomatous polyposis. Am $\mathrm{J}$ Ophthalmol 1998;125:680-6.

35. Soravia C, Berk T, McLeod RS, Cohen Z. Desmoid disease in patients with familial adenomatous polyposis. Dis Colon Rectum 2000;43:363-9.

36. Spigelman AD, Williams CB, Talbot IC, Domizio P, Phillips RK. Upper gastrointestinal cancer in patients with familial adenomatous polyposis. Lancet 1989;2:783.

37. Tiret A, Taiel-Sartral M, Tiret E, Laroche L. Diagnostic value of fundus examination in familial adenomatous polyposis. Br J Ophthalmol 1997;81:755-8.

38. Tsukada K, Church JM, Jagelman DG, Fazio VW, McGannon E, George CR, Schroeder T, Lavery I, Oakley J. Noncytotoxic drug therapy for intra-abdominal desmoid tumor in patients with familial adenomatous polyposis. Dis Colon Rectum 1992;35:29-33.

39. Turcot J, Despres JP, Pierre F. Malignant tumors of the central nervous system associated with familial polyposis of the colon: report of 2 cases. Dis Colon Rectum 1959;2:465-8.
40. Vellutini EA, Pahl FH, Vieira MJ, De Aguiar PH, Vellutini DF, De Almeida GM, Habr-Gama A. Turcot syndrome: a report of 2 cases. Arq Neuropsiquiatr 1990;48:102-6.

41. Wallis YL, MacDonald F, Hulten M, Morton JE, McKeown CM, Neoptolemos JP, Keighley M, Morton DG. Genotype-phenotype correlation between position of constitutional APC gene mutation and CHRPE expression in familial adenomatous polyposis. Hum Genet 1994;94:543-8.

42. Warde P, Canto AL, Cavalcante F, Habr-Gama A. Tumor maligno do sistema nervoso central associado à polipose do cólon (síndrome de Turcot). Apresentação de um caso. Arq Gastroenterol 1976;13:119-123.

43. Wexner SD, Jagelman DG. Familial polyposis syndromes. Sem Colon Rectal Surg 1991;2:269-76. 